\title{
Evidencias de la práctica Pilates sobre la salud mental de personas sanas
}

\author{
Evidence of Pilates practice on mental health of healthy people
}

Salvador Boix-Vilella1* ${ }^{*}$ orcid.org/0000-0002-7647-7767

Eva León-Zarceño ${ }^{2}$ orcid.org/0000-0003-1480-706X

Miguel Ángel Serrano-Rosa ${ }^{3}$ orcid.org/0000-0002-6574-4532

1 Departamento de Orientación, Instituto de Educación Secundaria Maciá Abela. Crevillent, España.

2 Departamento de Psicología de la Salud, Universidad Miguel Hernández de Elche. Elche, España

3 Departamento de Psicobiología, Universitat de Valencia. Valencia, España.

\begin{abstract}
Resumen
Introducción: El método de Pilates fue diseñado para crear una conexión entre la mente y el cuerpo, mediante un trabajo combinado de fuerza y flexibilidad, sin someter al cuerpo a una tensión excesiva. Objetivo: Exponer desde una perspectiva analítica-interpretativa los resultados de investigación más relevantes, en relación a la salud mental de personas sanas que practican habitualmente Pilates. Materiales y métodos: Se realizó un análisis de los principales beneficios del método Pilates sobre la salud mental, con resultados de investigaciones terminadas; para ello, se accedió a las bases de datos ScienceDirect, PubMed y Dialnet para identificar publicaciones que relacionan Pilates con variables mentales. Resultados: Los 19 estudios revisados muestran evidencias científicas sobre los niveles de humor, autoeficacia, autoestima, estado de ánimo, estrés, calidad de sueño, depresión e identificación con el ejercicio físico. Pero al analizar estudios centrados en la atención, ansiedad-estado, afecto positivo o negativo y calidad de vida; se comprueba que la evidencia es todavía muy limitada. Conclusiones: Son necesarios nuevos trabajos de corte longitudinal con muestras suficientemente amplias que permitan despejar las incógnitas aún existentes en relación a Pilates; esto permitirá determinar si la elevada popularidad del método se asocia con los importantes beneficios reportados sobre la salud.
\end{abstract}

Palabras clave: Método Pilates; ejercicio; salud mental; calidad de vida; depresión. (Fuente: DeCS, Bireme).

\begin{abstract}
Introduction: The Pilates method was designed to create a connection between mind and body through a combined work of strength and flexibility, without submitting the body to excessive stress. Objective: To expose the most relevant research results from an analytical-interpretative perspective, in relation to the mental health of healthy people who habitually practice Pilates. Materials and methods: An analysis of the main benefits of the Pilates method on mental health was carried out, by using the results of finished research. To do this, the databases ScienceDirect, PubMed and Dialnet were accessed to identify publications that relate Pilates to mental variables. Results: The 19 revised studies show scientific evidence on the levels of humor, self-efficacy, self-esteem, mood, stress, quality of sleep, depression and identification with physical exercise. However, studies focused on attention, anxiety-state, positive or negative affect and quality of life proved that the evidence is still very limited. Conclusions: New longitudinal cutting researches using sufficiently wide samples are necessary to clear the unknowns still
\end{abstract}


existing in relation to Pilates. This will determine whether the high popularity of the method is associated with the important health benefits reported.

Keywords: Pilates; exercise; mental health; quality of life; depression. (Source: DeCS, Bireme).

\section{Introducción}

Joseph Hubertus Pilates, creador del método Pilates, nació en 1880 en Mönchengladbach (Alemania). Desde su infancia se interesó por el estudio y la práctica de actividades físicas como el boxeo, esquí, danza y gimnasia. Tras la Primera Guerra Mundial, Joseph Pilates se trasladó de Alemania a Nueva York donde inauguró su primer estudio en el que consolida la contrología, que es el término con el que se refería a su método(1). La contrología se define como la ciencia y arte del desarrollo coordinado de la mente, el cuerpo y el espíritu, a través de movimientos naturales, bajo el estricto control de la voluntad(2). En esa misma línea, otros autores consideran que el objetivo del método Pilates es crear una conexión entre la mente y el cuerpo para que sin pensarlo, uno sea capaz de estar de pie, moverse y llevar a cabo las actividades diarias con control y facilidad(3). En cada movimiento se realiza un trabajo combinado y simultáneo de fuerza y flexibilidad, de manera que se habitúa al cuerpo a realizar un esfuerzo controlado en un amplio rango de movilidad articular, sin someter al cuerpo a una excesiva tensión(4).

El método se compone de más de 500 ejercicios que buscan el estiramiento y fortalecimiento de todos los músculos del cuerpo. La técnica Pilates trabaja el sistema muscular a través de cadenas musculares en lugar de músculos aislados(5). Los movimientos pueden realizarse en el suelo con ayuda de materiales e implementos, o en máquinas especialmente $\operatorname{diseñadas(6).~La~}$ modalidad mat Pilates o suelo desarrolla los ejercicios sólo en colchoneta, utilizando como resistencia el peso de propio cuerpo. En cambio, los ejercicios de máquinas se llevan a cabo en aparatos desarrollados por el propio Pilates(1). El número de principios que rigen la práctica del método y el orden de los mismos varían según el autor, aunque los principios comunes en la mayoría de escuelas de Pilates son: concentración, centralización, control, respiración, precisión y fluidez ${ }^{(7)}$. El Pilates clásico u original se refiere a la realización de ejercicios de contrología en el orden formulado por Joseph Pilates y con la columna en posición neutral(8). La forma en que se realizan es más importante que los mismos ejercicios(3). Actualmente, existen nuevas modalidades como por ejemplo el aeroPilates, el Pilates power plate o el yogalates que incorporan cambios a los principios marcados por Joseph Pilates y que no cuentan con el respaldo científico necesario para considerarlas método Pilates original( ${ }^{(9)}$.

Se dice que Pilates fue un auténtico pionero o adelantado en su tiempo al ser de los primeros occidentales en estudiar diferentes disciplinas orientales como el Yoga o el Zen, hasta el punto que muchos catalogan su método como el yoga occidental(10). Las relaciones del Pilates con el yoga e incluso el taichí son más que evidentes ya que dichas actividades combinan la vertiente deportiva con el bienestar corporal, mental y social(11). Estas conexiones con actividades más consolidadas, y por tanto más conocidas en la sociedad, han podido favorecer su aceptación. Además, el método Pilates es un tipo de actividad física muy enfocada al mantenimiento y la mejora de salud por cuanto se adapta perfectamente a las nuevas demandas que solicitan más actividades cuerpo-mente, ejercicio consciente y el trabajo postural(9,12). De hecho, actualmente, la técnica Pilates ya cuenta con millones de practicantes en todo el mundo(13).

Conscientes de los numerosos beneficios que genera el ejercicio físico, los investigadores cada vez se muestran más interesados en analizar los efectos directos del ejercicio sobre la salud(14). Sin embargo, la dificultad para desarrollar estudios experimentales de causa-efecto, hace que los resultados del ejercicio sobre la salud física estén mejor establecidos que sobre el bienestar psicológico o social(15). En este sentido, el actual concepto de salud se refiere a un estado 
completo de bienestar físico, mental y social(16). En el caso de la salud mental, el yoga aparece en los primeros escritos encontrados como una técnica que desde un punto de vista físico buscaba mejorar las funciones mentales(17). En el caso concreto del método Pilates, las primeras investigaciones que se pueden encontrar en la base de datos científica MEDLINE con la palabra clave "Pilates" datan del año 1999. Años más tarde, concretamente en el 2004, se publica el primer estudio de intervención donde se señala la dificultad de establecer los efectos del método sobre el estado de salud(18). Retomando el concepto de salud mental, la conexión cuerpomente que promulga el Pilates podría aportar experiencias positivas a los practicantes que buscan mejorar su bienestar psicológico(19). El modelo estructural del impacto del Pilates sobre los factores psicológicos considera que este tipo de actividad afecta de manera diferente a cada factor, considerando cada uno de los casos por separado(20). A pesar de este primer modelo teórico sobre Pilates, sigue existiendo poca evidencia científica sobre personas sanas ya que la mayoría de las investigaciones en el contexto psicosocial se han llevado a cabo con participantes de edad avanzada o con personas que presentaban diferentes patologías médicas(21).

En las bases de datos analizadas el número de publicaciones aumenta progresivamente. Ahora bien, exceptuando mejoras en la salud física del practicante, fácilmente constatables, se evidencia la necesidad de nuevos trabajos sobre Pilates centrados en el análisis de la salud psicológica y social(9). A pesar de ello, un reciente estudio ha expuesto el mecanismo por el cual el Pilates podría ayudar a mejorar la salud mental, concretamente reduciendo los niveles de ansiedad en estudiantes( ${ }^{(20)}$. Según éste trabajo, la práctica de Pilates en su conjunto aumenta la fuerza corporal, la calidad del sueño, la atención, la concentración, el flujo sanguíneo, la circulación de oxígeno en el cerebro, aleja los pensamientos negativos, el cuerpo se siente más cómodo, los músculos están más relajados y todo ello junto a una adecuada respiración ayudaría a reducir la ansiedad.
En función de los antecedentes presentados, se plantea como objetivo exponer desde una perspectiva analítica-interpretativa los resultados de investigaciones más relevantes en relación a la salud mental de personas sanas que practican habitualmente Pilates.

\section{Materiales y métodos}

La investigación es de tipo cualitativa con un claro componente descriptivo. A partir de los resultados de investigación terminada, se lleva a cabo una búsqueda en las bases de datos científicas ScienceDirect, PubMed y Dialnet. La palabra "Pilates" siempre estuvo presente en uno de los campos de búsqueda. Además, se emplearon los descriptores "salud psicológica" y "salud mental" en español y en inglés. Las búsquedas se realizaron hasta el día 25 de mayo de 2017. Respecto a los criterios de exclusión, se establecen: a) estudios escritos en idioma distinto al español o al inglés; b) estudios centrados en la salud física del practicante; c) comunicaciones cortas y estudios científicos publicados en forma de resumen; d) estudios que no utilizan el método Pilates como variable diferenciadora frente a otras técnicas de ejercicio físico; e) trabajos que pese a emplear la técnica Pilates como variable diferenciadora estudian a participantes diagnosticados con dolencias físicas o psíquicas; f) estudios que incluyan a participantes mayores de 65 años por cuanto los autores del presente estudio consideran que la condición de salud se encuentra debilitada en mayor o menor grado como consecuencia de la aparición de posibles síntomas que anuncian la disfunción de órganos y sistemas.

Una vez detectados los estudios que analizaban los beneficios del Pilates sobre la salud mental de las personas sanas, se leyeron los trabajos en toda su extensión. En el caso de los estudios incluidos que analizaban una combinación de variables físicas y mentales se omitieron aquellos resultados que no tenían relación directa con el objetivo del presente trabajo. 


\section{Resultados}

Durante las últimas dos décadas se ha venido produciendo un progresivo incremento en el número de trabajos científicos que estudian Pilates (Figura 1). Sin embargo, sigue siendo una modalidad de entrenamiento poco investigada en comparación con otros ejercicios físicos como el Yoga o el Taichí. En el caso concreto de la base de datos ScienceDirect, se registran, sólo en el año 2016, un total de 1063 trabajos con la palabra clave "yoga" y un total de "948" con la palabra clave "taichí” frente a los 156 del Pilates.

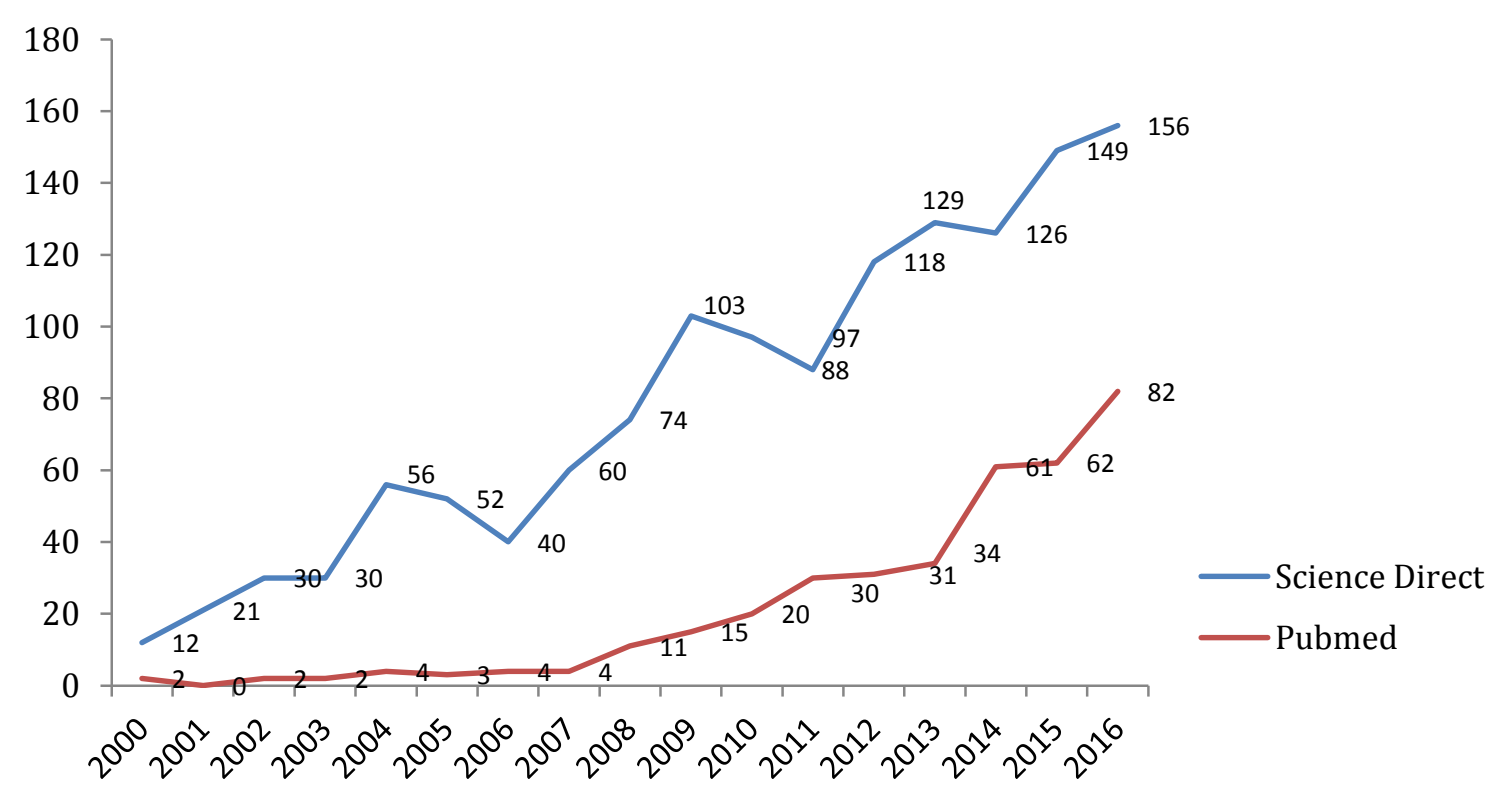

Figura 1. Número de publicaciones con la palabra clave "Pilates"

Tras aplicar los criterios de exclusión se revisó un total de 19 trabajos. Los resultados se han agrupado y expuesto en función de las características de los participantes que conformaban los diferentes estudios. De esta manera, se distingue entre estudiantes, mujeres, personas sedentarias y trabajadores en activo. Además, se exponen algunas conclusiones de los dos trabajos de revisión incluidos.

Un estudio realizado con estudiantes universitarios que practicaron Pilates durante seis meses mostró un aumento significativo del humor, de la autoeficacia y una tendencia a la mejora en la calidad del sueño(22). Posteriormente, se concluye que 15 sesiones de Pilates mejoran el estrés, la atención y el estado de ánimo de universitarios(23). En estudiantes de danza, un trabajo cuasi-experimental evidenció mejoras en la atención plena tras 15 semanas de práctica de Pilates(24). Un trabajo transversal con universitarios españoles comprobó que la ausencia de diferencias significativas en los niveles de ansiedad estado y rasgo entre un grupo Pilates y un grupo sedentario(25). Un estudio reciente en universitarias determina que la práctica de Pilates durante 10 semanas mejora significativamente el afecto negativo $y$ no encuentra cambios en los niveles de atención plena(21).

En mujeres de mediana edad, un ensayo controlado aleatorio con una duración de seis meses revela mejoras significativas en las variables: satisfacción con la vida, percepción de apreciación de personas y apariencia(26). Un segundo ensayo clínico aleatorizado, esta vez con mujeres tras dar a luz, comprueba que ocho semanas de Pilates son suficientes para mejorar la calidad del sueño frente a otro grupo de 
mujeres control(27). En un estudio prospectivo, con mujeres menopaúsicas de edades comprendidas entre los 38 y 63 años, se demostró que 12 semanas consecutivas de Pilates mejoraron la salud mental, el rol emocional y la función social(28). A pesar de ello, éstos autores reclaman nuevos estudios con muestras más grandes y con un seguimiento más largo para poder evaluar si los resultados positivos se mantienen. Tras un programa de ocho semanas de Pilates con mujeres de 20 a 45 años, se encontraron mejoras en las puntuaciones referentes a depresión y autoestima obtenidas por el grupo experimental frente a otro grupo control(29). Un estudio centrado en la variable depresión de mujeres premenopáusicas muestra una disminución de la sintomatología depresiva tras 12 semanas de Pilates(30).

En el caso de personas sedentarias, un primer trabajo llevó a cabo un programa de 12 semanas con Pilates y se obtuvo mejoras en la calidad del sueño y en la calidad de vida(31). Un segundo estudio prospectivo en personas sedentarias de mediana edad, mostró mejoras en componentes emocionales del cuestionario de calidad de vida (SF-36) y en la latencia y calidad del sueño tras 12 semanas practicando Pilates ${ }^{(32)}$. Un trabajo de seguimiento, centrado en determinar el efecto del desentrenamiento en mayores de 30 años tras un programa de Pilates y tres meses de inactividad, señala al grupo Pilates como el que obtiene mayores puntuaciones aunque no se detectaron diferencias significativas intergrupos ni intragrupo en la variable calidad de vida(33).

En relación a las personas que se encuentran trabajando, la práctica de Pilates se asocia con mayores niveles de identificación con el ejercicio físico lo que aumenta la probabilidad de permanecer durante más tiempo activo(34). Un reciente estudio descriptivo-comparativo, centrado en la salud psicosocial de trabajadores que venían practicando Pilates habitualmente, muestra en la medición inicial la existencia de mayores niveles de estabilidad emocional y optimismo que un grupo sedentario, aunque tras seis meses de seguimiento se evidencia la ausencia de importantes diferencias en la salud psicosocial y laboral(35). Este mismo estudio señala que los directivos presentan mayor control en el trabajo, mayor sintomatología depresiva y menor estabilidad emocional que los empleados en ambas mediciones, al inicio y tras seis meses de estudio. Por último, una tesis doctoral centrada en la salud psicosocial y laboral concluyó que las personas que se ejercitan con Pilates, tras cuatro meses de seguimiento con escalas estado, reducen sus niveles de afecto negativo, de ansiedad-estado y de afecto positivo. Además, a largo plazo, tras 5 o más años de práctica de Pilates, las personas obtienen mayores niveles de autoconcepto e identificación con el ejercicio físico en relación a otras más inexpertas ${ }^{(9)}$.

Una primera revisión centrada en la efectividad del método Pilates en personas sanas muestra evidencia limitada en variables como la satisfacción con la vida, el autoconcepto y la percepción del estado de salud(36). También señala la ausencia de evidencias en autoeficacia, estado de ánimo, calidad del sueño, equilibrio mental y calidad de vida. Una revisión más reciente centrada en los efectos del Pilates en la salud psicosocial, que incluye estudios con personas sanas y con diferentes patologías, señala la necesidad de nuevos trabajos con una mejor metodología y con muestras más amplias para dilucidar los efectos reales del Pilates(37). Por último se destaca la existencia de un estudio que muestra mayores niveles de identificación con el ejercicio físico entre los practicantes de Pilates( ${ }^{(38)}$.

\section{Discusión y conclusiones}

Tras la revisión llevada a cabo, para dar respuesta al objetivo de investigación, se constata la dificultad existente para realizar estudios longitudinales suficientemente duraderos con una muestra amplia ${ }^{(9,39)}$. De los 19 estudios revisados, 15 de ellos son considerados de intervención y emplean una duración media de 15,73 semanas de Pilates. Estos resultados señalan que existe una preferencia entre los investigadores por los trabajos de corte longitudinal ya que sólo dos transversales han sido incluidos en el presente estudio(25,34). Sin 
embargo, el tamaño muestral en los estudios de Pilates suele ser pequeño como se observa en los

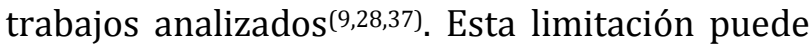
deberse a que las agrupaciones en Pilates suelen ser flexibles, variando el día o la hora de la sesión que mejor se adapta a las necesidades de los alumnos, y afectando de manera importante al registro y seguimiento del investigador, disminuyendo por tanto el número de sujetos que completan el estudio(9). Por tanto, a las dificultades ya conocidas para el estudio de variables mentales hay que añadir la elevada muerte experimental que se da al analizar el Pilates.

A pesar de las dificultades, en estudiantes, se detectan las primeras evidencias en el humor, la autoeficacia, el estado de ánimo, el estrés y el afecto negativo. Las mejoras obtenidas en el humor y en el estado de ánimo son consideradas el tercer motivo de práctica de Pilates, en hombres y en mujeres, tras la mejora de la postura corporal y la flexibilidad(40). En el caso de la atención plena, los resultados son contradictorios, en los estudios analizados, y no se encuentran evidencias significativas en relación a la ansiedad rasgo-estado ni en la calidad del sueño. En total son cinco el número de trabajos que relacionan variables mentales y Pilates en estudiantes, lo que supone el $29,41 \%$ de los estudios experimentales incluidos. Éste elevado interés podría deberse a la accesibilidad que tiene el personal investigador universitario a estudiantes de su propia universidad.

En relación a las mujeres, se encuentran mejoras en la calidad del sueño tras dar a luz, en el rol emocional, la función social, la depresión, la autoestima, la satisfacción con la vida, la percepción de apreciación por otras personas y la apariencia. De los 15 estudios analizados, el $46,67 \%$ se centra exclusivamente en mujeres y el $53,33 \%$ restante está formado por hombres y mujeres. La abrumadora presencia de la mujer adulta en la investigación de Pilates podría deberse a que representan el porcentaje mayoritario en las clases de Pilates, al ser una actividad con un bajo componente aeróbico y que está muy enfocada a la mejora de la salud.
Respecto a las personas sedentarias que inician un programa de 12 semanas de Pilates, se observan mejoras en la calidad del sueño y de vida en los dos trabajos incluidos(31,32). En relación al desentrenamiento, parece que los niveles de calidad de vida no se ven influenciados por la actividad física practicada. Por último, en el caso de trabajadores que incluyen la técnica Pilates como método de entrenamiento, se señala que no hay cambios importantes en los niveles de salud psicosocial y laboral después de seis meses de seguimiento. Sin embargo, tras 5 o más años de práctica continuada de Pilates, las personas obtienen mayores niveles de autoconcepto e identificación con el ejercicio físico en relación a otros participantes de Pilates más inexpertos.

A pesar de que la evidencia científica del Pilates en la salud mental de personas sanas es todavía limitada no se puede obviar que es una técnica que cuenta con millones de participantes en todo el mundo. El hecho de que famosos internacionales del mundo de la canción y del cine reconozcan abiertamente practicar Pilates para mejorar su rendimiento y ampliar sus carreras, puede haber ayudado a aumentar su práctica fuera de las fronteras de Estados Unidos(41). Además, deportistas del mundo del tenis, del golf y plantillas de fútbol, emplean Pilates como complemento a su preparación, otorgándole de forma indirecta mayor importancia a la práctica de Pilates(42). Conscientes de este crecimiento, cada vez son más las clínicas de fisioterapia y los centros de Fitness que ofrecen a sus clientes la posibilidad de practicar Pilates, favoreciendo el acceso del método a multitud de grupos poblacionales ${ }^{(9)}$.

Como consecuencia de las nuevas variedades existentes de Pilates, las futuras publicaciones deberían concretar con la mayor precisión posible el apartado metodológico, definiendo con exactitud la técnica Pilates estudiada. Además, sería interesante el análisis de las dos modalidades del Pilates original: máquinas y suelo, por separado y combinadas. Desde el presente trabajo, se recomienda que los nuevos estudios sean de corte longitudinal, que empleen muestras amplias y lleven a cabo mediciones 
antes, durante y después de la intervención con el método Pilates.

Los autores del presente estudio desconocen si con el tiempo esta actividad perderá importancia y será relegada por otras actividades más novedosas. De lo que sí están convencidos es que la comunidad científica, ante las necesidades expuestas en éste y otros trabajos, debe seguir analizando los beneficios reales que aporta a la salud mental la práctica de Pilates en todas sus versiones, descartando que los beneficios encontrados no sean fruto de un mayor grado de apoyo social y de un estilo de vida saludable como consecuencia de participar en un programa de actividad física. Los planteamientos aquí formulados para la práctica de Pilates podrían hacerse extensibles a otras actividades como el Yoga o el Taichí.

\section{Conflicto de intereses}

Los autores del presente trabajo declaran la ausencia de cualquier conflicto de intereses.

\section{Referencias}

1. Latey P. The Pilates method: History and philosophy. Journal of Bodywork and Movement Therapies. 2001;5(4):275-82.

2. Pilates J. Your health. Incline Village, Nevada: Presentation Dynamics Incorporated; 1934.

3. Nealy B, Cooper C. Applying Pilates concepts to hand therapy: Connecting through the hand. Fundamentals of Hand Therapy. 2014;2:192-96.

4. García-Pastor T. Efecto de la práctica del método Pilates: Beneficios en estado de salud, aspectos físicos y comportamentales. Toledo: Universidad de Castilla la Mancha; 2009.

5. Cárceles R, Cos F. Manual completo de Pilates suelo. Barcelona: Paidotribo; 2009.

6. Hernández L, Gómez M, Carrasco M, Baena A. El Pilates como alternativa saludable a los contenidos tradicionales de las clases de Educación Física en Primaria. Espiral Cuadernos del Profesorado. 2011;4(8):77-92.

7. McNeill W. The double knee swing test a practical example of the performance matrix movement screen. Journal of Bodywork and Movement Therapies. 2014;18(3):477-81.

8. Shea S, Moriello G. Feasibility and outcomes of a classical Pilates program on lower extremity strength, posture, balance, gait, and quality of life in someone with impairments due to a stroke. Journal of Bodywork and Movement Therapies. 2014;18(3):332-60.
9. Boix S. Efectos de la práctica del método Pilates sobre la salud psicosocial: Un estudio longitudinal. Elche: Universidad Miguel Hernández; 2016.

10. Fernández E, Santana F, Merino R. Joseph Hubertus Pilates: Anatomía de un gigante olvidado. Trances. 2011;3(3):353-78.

11. Serrano MA, Boix S. Efectos del tipo y cantidad de actividad física en la salud psicológica percibida de profesoras. Revista Iberoamericana de Psicología del Ejercicio y el Deporte. 2012;7(1):149-61.

12. Muirhead M. Total Pilates. Madrid: Pearson; 2004.

13. Boix S, León E, Serrano M. Apoyo social y bienestar psicológico en mujeres practicantes de Pilates. In: Soler J, Aparicio L, Díaz 0, Escolano E, Rodríguez A, editors. Inteligencia emocional y Bienestar II Reflexiones, experiencias profesionales e investigaciones. Villanueva de Gallego: Ediciones Universidad de San Jorge; 2016. p. 394-404.

14. Queirós $M$, Carral J, Fernández-Berrocal P. Inteligência emocional percebida e actividade física na terceira idade. Revista de Psicologia, Educação e Cultura. 2004;8(1):187-209.

15. Jiménez M, Martínez P, Miró E, Sánchez A. Bienestar psicológico y hábitos saludables: ¿Están asociados a la práctica de ejercicio físico? International Journal of Clinical and Health Psychology. 2008;8(1):185-202.

16. Organización Mundial de la Salud. Constitution of the World Health Organization. Handbook of basic documents. Geneva: Palais des Nations; 1952.

17. Catalán D. Fisioterapia en salud mental. Efectividad de una intervención fisioterapéutica en trastornos alimentarios. Málaga: Universidad de Málaga; 2007.

18. Segal N, Hein J, Basford J. The effects of Pilates training on flexibility and body composition: An observational study. Archives of Physical Medicine and Rehabilitation. 2004;85(12):1977-81.

19. De Souza M, Vieira C. Who are the people looking for the Pilates method? Journal of Bodywork and Movement Therapies. 2006;10(4):328-34.

20. Memmedova K. Impact of Pilates on anxiety attention, motivation, cognitive function and achievement of students: Structural modeling. Social and Behavioral Sciences. 2015;186:544-8.

21. Tolnai N, Szabó Z, Köteles F, Szabo A. Physical and psychological benefits of once-a-week Pilates exercises in young sedentary women: A 10-week longitudinal study. Physiology \& Behavior. 2016;163:211-8.

22. Caldwell K, Harrison M, Adams M, Triplett N. Effect of Pilates and taiji quan training on self-efficacy, sleep quality, mood, and physical performance of college students. Journal of Bodywork and Movement Therapies. 2009;13(2):155-63.

23. Caldwell K, Harrison M, Adams M, Quin R, Greeson J. Developing mindfulness in college students through movement-based courses: Effects on self-regulatory, self-efficacy, mood, stress, and sleep quality. The journal of American College Health. 2010;58(5):433-42.

24. Caldwell K, Adams M, Quin R, Harrison M, Greeson J. Pilates, mindfulness and somatic education. Journal of Dance \& Somatic Practices. 2013;5(2):141-53. 
25. Herrera-Gutiérrez E, Olmos M, Brocal D. Efectos psicológicos de la práctica del método Pilates en una muestra universitaria. Anales de Psicología. 2015;31(3):916-20.

26. Cruz-Ferreira A, Fernandes J, Gomes D, Bernardo L, Kirkcaldy B, Barbosa T, et al. Effects of Pilates-based exercise on life satisfaction, physical self-concept and health status in adult women. Women and Health. 2011;51(3):240-55.

27. Ashrafinia F, Mirmohammadali M, Rajabi $H$, Kazemnejad A, Sadeghniiathaghighi K, Amelvalizadeh $M$, et al. The effects of Pilates exercise on sleep quality in postpartum women. Journal of Bodywork and Movement Therapies. 2014;18(2):190-9.

28. Rodríguez-Fuentes G, Machado I, Ogando-Berea $\mathrm{H}$, Otero-Gargamala M. An observational study on the effects of Pilates on quality of life in women during menopause. European Journal of Integrative Medicine. 2014;6(6):631-6.

29. Küçük F, Livanelioflu A. Impact of the clinical Pilates exercises and verbal education on exercise beliefs and psychosocial factors in healthy women. Journal of Physical Therapy Science. 2015;27(11):3437-43.

30. Halis F, Yildirim P, Kocaaslan R, Cecen k, Gokce A. Pilates for better sex: Changes in sexual functioning in healthy Turkish women after Pilates exercise. Journal of sex \& Marital Therapy. 2015;42(4):302-8.

31. Leopoldino A, Avelar N, Passos GB, Santana N, Teixeira $V$, De Lima V, et al. Effect of Pilates on sleep quality and quality of life of sedentary population. Journal of Bodywork and Movement Therapies. 2013;17(1):5-10.

32. García-Soidán J, Giraldez V, Cachón-Zagalaz A, LaraSánchez A. Does Pilates exercise increase physical activity, quality of life, latency and sleep quantity in middle aged people? Perceptual and Motor Skills. 2014;119(3):838-50.

33. Cáscales-Ruiz E, Del Pozo-Cruz J, Alfonso-Rosa R. Efectos de 12 semanas de desentrenamiento en la retención de la condición física y calidad de vida en mujeres mayores de 30 años tras un programa de nueve meses de Pilates y comparación con mujeres sedentarias. Revista española de Educación Física y Deportes. 2015;408:23-37.

34. Boix S, León E, Serrano M. Niveles de identificación con el ejercicio físico en una muestra de profesores que se ejercita con Pilates. In: Castejón J, editor. Psicología y Educación: Presente y futuro. Madrid: ACIPEAsociación Científica de Psicología y Educación; 2016. p. 2751-6.

35. Boix S, León E, Serrano M. Salud psicosocial en trabajadores que practican Pilates: Un estudio descriptivo-comparativo. Cultura, Ciencia y Deporte. 2017;12(34):27-37.

36. Cruz-Ferreira A, Fernandez J, Laranjo L, Silva A. A systematic review of the effects of Pilates method of exercise in healthy people. Archives of Physical Medicine and Rehabilitation. 2011;92(12):2071-81.

37. Boix S, León E, Serrano M. ¿Hay beneficios psicosociales por la práctica Pilates? Un análisis de la literatura científica. Cuadernos de Psicología del Deporte. 2014;14(3):117-28.
38. Boix S, León E, Serrano M. Identificación con el ejercicio físico y autoeficacia: Diferencias entre practicantes de Pilates vs no practicantes. Revista Iberoamericana de Psicología del Ejercicio y el Deporte. 2017;12(1):99106.

39. Bernardo L. The effectiveness of Pilates training in healthy adults: An appraisal of the research literature. Journal of Bodywork and Movement Therapies. 2007;11(2):106-10.

40. León E, Boix S, Serrano M. Optimismo, Identificación con el ejercicio físico y motivos de práctica de Pilates: Diferencias de sexo. Revista Cubana de Medicina del Deporte y la Cultura Física. 2016;11(3):1-16.

41. Isacowitz R. Pilates. Manual completo del método Pilates. Barcelona: Paidotribo; 2009.

42. Del Olmo N. Pilates como complemento del entrenamiento en fútbol de alto rendimiento. Madrid: Universidad Politécnica de Madrid; 2015. 